\title{
Publisher Correction: Perovskites take steps to industrialization
}

Correction to: Nature Energy https://doi.org/10.1038/s41560-020-0552-6, published online 22 January 2020.

In the version of this Editorial originally published, the name 'Eugene Katz' was spelt incorrectly as 'Eugene Kats'. This has now been corrected in all versions.

Published online: 28 January 2020

https://doi.org/10.1038/s41560-020-0564-2

(c) Springer Nature Limited 2020 